\title{
ARTICLE \\ Composite particles with magnetic properties, near-infrared excitation, and far-red emission for luminescence-based oxygen sensing
}

\author{
Elisabeth Scheucher ${ }^{1}$, Stefan Wilhelm ${ }^{2}$, Otto S. Wolfbeis ${ }^{2}$, Thomas Hirsch ${ }^{2}$ and Torsten Mayr ${ }^{1}$
}

Oxygen sensing, magnetic, and upconversion luminescence properties are combined in multi-functional composite particles prepared herein by a simple mixing, baking, and grinding procedure. Upconverting nanocrystals are used as an excitation source and an oxygen indicator with far-red emission. The composite particles are excited with near infrared (NIR) laser light ( $980 \mathrm{~nm}$ ). The visible upconversion emission is converted into an oxygen concentration-dependent far-red emission $(<750 \mathrm{~nm})$ using an inert mediator dye and a platinated benzoporphyrin dye. This concept combines the advantages of NIR excitation and far-red emissive indicator dyes, offering minimized auto-fluorescence and enhanced membrane permeability. Additional functionality is obtained by incorporating magnetic nanoparticles into the composite particles, which enables easy manipulation and separation of the particles by the application of an external magnetic field.

Keywords: magnetic particles; optical sensor; oxygen sensor; upconverting nanoparticles

Microsystems \& Nanoengineering (2015) 1, 15026; doi:10.1038/micronano.2015.26; Published online: 19 October 2015

\section{INTRODUCTION}

Oxygen is essential for nearly all living systems, thus it is one of the most important chemical species on earth. Knowledge of the actual oxygen concentration is of particular significance in areas such as medical diagnostics, biology, biotechnology, marine research, and environmental analysis ${ }^{1}$. In recent years, optical chemical sensors have proven to be valuable and flexible tools for online monitoring and control of analyte concentrations ${ }^{2}$. Optical sensor systems for oxygen are typically based on gas-permeable polymer matrices containing oxygen-sensitive probes. Such a design allows for the fabrication of sensor films or spots, which can be placed inside any optical transparent vessel, enabling a contactless and non-invasive readout for subsequent data processing and further analysis ${ }^{3}$. Another approach is the application of particles with micro- or nanometre dimensions and oxygensensing capabilities ${ }^{4-6}$. Such particles combine the benefits of sensor layers with the flexibility of dissolved indicators. Nanoparticles can easily be applied by adding them to a corresponding measurement setup or reaction chamber ${ }^{7-9}$. A promising class of materials for sensor development is upconverting crystals because of their near-infrared (NIR) excitation.

Lanthanide-doped inorganic upconverting luminescent nanoparticles (UCLNPs) are capable of emitting visible light upon NIR excitation ${ }^{10}$. UCLNPs consisting of hexagonal-phase $\mathrm{NaYF}_{4}$ acting as a crystalline host material and ytterbium(III) and erbium(III) dopants as sensitizer and activator ions, respectively, are the most efficient upconverting phosphors known to date ${ }^{11}$. The generation of upconversion luminescence follows a photophysical process called energy transfer upconversion ${ }^{12}$. The excitation energy (usually at a wavelength of $980 \mathrm{~nm}$ ) is absorbed by $\mathrm{Yb}^{3+}$ sensitizers and then sequentially transferred to $\mathrm{Er}^{3+}$ activators, which results in anti-Stokes emission. These emission bands are fairly narrow with a full-width-at-half-maximum (FWHM) typically less than $20 \mathrm{~nm}$. In contrast to simultaneous two-photon absorption, which requires expensive high-power femtosecondpulsed lasers, UCLNPs are excited by low-cost, low-power continuous wave (CW) laser diodes. NIR excitation causes minimal photo-damage to biological specimens and does not excite endogenously fluorescent moieties in biological matter.

These advantageous features along with excellent photostability render UCLNPs highly attractive for bioimaging and bioanalytical applications ${ }^{13-16}$. Recently, UCLNPs have been employed as nanolamps for monitoring enzymatic reactions and for the determination of glucose and ethanol levels ${ }^{17,18}$. Optical chemical sensor films for $\mathrm{pH}$, ammonia, $\mathrm{CO}_{2}$, cyanide anions, or metal ions have also been reported ${ }^{19-23}$. Very recently, UCLNPs with sensingfunctionalities for $\mathrm{pH}$, mercury ions, and temperature were demonstrated $^{24-27}$.

Herein, we present multifunctional particles, which combine oxygen sensing, magnetic, and upconversion luminescence properties in one unit. The sensing functionality enables the quantitative detection of gaseous and dissolved oxygen. In contrast to other sensor systems based on upconversion luminescence mainly using green or red emission, we employ an oxygen sensitive, far-red/NIR emitting platinum(II)tetraphenyltetrabenzoporphyrin (PtTPTBP) dye. This dye facilitates penetration of the emitted light from biological materials attributed to the reduced scattering compared to green and red light. The magnetic functionality enables easy manipulation and separation of these particles from the sample, as well as control over their concentration at particular positions by applying an external magnetic field ${ }^{28}$. Recently, the application of magnetic sensor particles without upconversion capabilities as flexible analytical tools in microfluidic devices was reported by our group ${ }^{29}$. The present sensing scheme based on the utilization of UCLNPs as

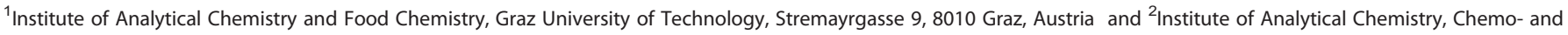
Biosensors, University of Regensburg, 93040 Regensburg, Germany

Correspondence: Torsten Mayr (torsten.mayr@tugraz.at)

Received: 1 July 2015; revised: 18 August 2015; accepted: 26 August 2015 
nanolamps for indirect excitation of an oxygen-quenchable probe minimizes autofluorescence from the sample matrix.

\section{MATERIALS AND METHODS}

\section{Materials}

Yttrium(III) chloride hexahydrate (99.99\%), ytterbium(III) chloride hexahydrate (99.9\%), erbium(III) chloride hexahydrate (99.9\%), ammonium fluoride (ACS reagent $\geq 98.0 \%$ ), and sodium hydroxide (reagent grade $\geq 98.0 \%$ ) purchased from Sigma-Aldrich (St. Louis, MO, USA, www.sigmaaldrich.com); oleic acid (technical grade 90\%) and 1-octadecene (technical grade 90\%) purchased from Alfa Aesar (Ward Hill, MA, USA, www.alfa.com); poly(phenylsilsesquioxane) (PPSQ), 100\% phenyl, 4.5-6.5\% OH purchased from ABCR (Karlsruhe, Germany, www.abcr.de); Macrolex fluorescent red G (MFR) purchased from Simon und Werner GmbH (Flörsheim am Main, Germany, www.simon-und-werner.de); EMG1300 purchased from FerroTec (Santa Clara, CA, USA, www.ferrotec.com); ethanol and chloroform (technical grade 90\%) purchased from VWR (Radnor, PA, USA, www.vwr.com); nitrogen 5.0 purchased from Air Liquide (Paris, France, www.airliquide.com); and osmosis water and compressed air were used.

PtTPTBP was synthesized by our group ${ }^{30}$. All other reagents and organic solvents were of the highest grade available.

\section{Synthesis of hydrophobic $\mathrm{NaYF}_{4}$ nanoparticles doped with $\mathrm{Yb} / \mathrm{Er}$}

Hydrophobic, lanthanide-doped $\mathrm{NaYF}_{4}$ nanocrystals were prepared via a modified procedure as reported by Zhang et al. ${ }^{31}$ $\mathrm{YCl}_{3} \cdot 6 \mathrm{H}_{2} \mathrm{O}(0.78 \mathrm{mmol}), \mathrm{YbCl}_{3} \cdot 6 \mathrm{H}_{2} \mathrm{O}(0.20 \mathrm{mmol})$, and $\mathrm{ErCl}_{3} \cdot 6 \mathrm{H}_{2} \mathrm{O}$ $(0.02 \mathrm{mmol})$ were used for the synthesis, which were dissolved in approximately $5 \mathrm{~mL}$ of methanol by sonication. The resulting clear and optically transparent solution of rare earth chlorides in methanol was transferred into a $50 \mathrm{~mL}$ flask, mixed with $8 \mathrm{~mL}$ of oleic acid and $15 \mathrm{~mL}$ of 1-octadecene under an atmosphere of nitrogen and heated to $160^{\circ} \mathrm{C}$. A homogeneous, clear solution was formed after $30 \mathrm{~min}$ at $160^{\circ} \mathrm{C}$ under vacuum. The reaction mixture was then cooled to room temperature and $10 \mathrm{~mL}$ of methanol containing $\mathrm{NaOH}(0.25 \mathrm{M})$ and $\mathrm{NH}_{4} \mathrm{~F}(0.4 \mathrm{M})$ was added immediately. The colloidal dispersion was heated to $120^{\circ} \mathrm{C}$ and stirred for $30 \mathrm{~min}$. The resulting colloid was refluxed at approximately $325^{\circ} \mathrm{C}$ for $15 \mathrm{~min}$. After cooling to room temperature, the UCLNPs were precipitated by addition of approximately $20 \mathrm{~mL}$ of ethanol and isolated via centrifugation at a relative centrifugal force (RCF) of $1000 \mathrm{~g}$ for $5 \mathrm{~min}$. The pellet was washed several times by dispersing it in small amounts (approximately $0.5 \mathrm{~mL}$ ) of chloroform and cyclohexane, followed by precipitation by the addition of a large excess (approximately $15 \mathrm{~mL}$ ) of ethanol and acetone. A white solid was obtained, which could easily be re-dispersed in cyclohexane to form a clear dispersion.

Synthesis of the $\mathrm{NaYF}_{4}$ shell $\mathrm{YCl}_{3} \cdot 6 \mathrm{H}_{2} \mathrm{O}(1 \mathrm{mmol})$ was dissolved in approximately $5 \mathrm{~mL}$ of methanol by sonication in a $50 \mathrm{~mL}$ flask, and mixed with $8 \mathrm{~mL}$ of oleic acid and $15 \mathrm{~mL}$ of 1 -octadecene. The solution was heated to $160{ }^{\circ} \mathrm{C}$ under an atmosphere of nitrogen. A homogeneous, clear solution was formed after $30 \mathrm{~min}$ at $160{ }^{\circ} \mathrm{C}$ under vacuum. The reaction mixture was then cooled to $80^{\circ} \mathrm{C}$. UCLNPs (ytterbium/erbium (Yb/Er) or ytterbium/thulium (Yb/Tm)) dispersed in cyclohexane were then added and the cyclohexane was evaporated. The mixture was cooled to room temperature and then $10 \mathrm{~mL}$ of methanol containing $\mathrm{NaOH}(0.25 \mathrm{M})$ and $\mathrm{NH}_{4} \mathrm{~F}(0.4 \mathrm{M})$ was added immediately. The colloidal dispersion was heated to $120^{\circ} \mathrm{C}$ and stirred for $30 \mathrm{~min}$ under an atmosphere of nitrogen. The resulting colloid was refluxed at approximately $325^{\circ} \mathrm{C}$ for $15 \mathrm{~min}$. After cooling to room temperature, the UCLNPs were precipitated by the addition of approximately $20 \mathrm{~mL}$ of ethanol and isolated via centrifugation at a RCF of $1000 \mathrm{~g}$ for $5 \mathrm{~min}$. The pellet was washed several times by dispersing in small amounts (approximately $0.5 \mathrm{~mL}$ ) of chloroform and cyclohexane, followed by precipitation by the addition of a large excess (approximately $15 \mathrm{~mL}$ ) of ethanol and acetone. A white solid was obtained, which could be easily redispersed in cyclohexane to form a clear dispersion. The size of the obtained monodisperse, hexagonal crystals was approximately 40 $\mathrm{nm}$, which was verified by transmission electron microscopy (TEM) images.

\section{Preparation of sensor particles}

Typically, $100 \mathrm{mg}$ of PPSQ was dissolved in $500 \mu \mathrm{L}$ of chloroform. Then, $10 \mathrm{mg}$ of UCLNPs and $0.5 \mathrm{mg}$ of organic dyes were added and the mixture was homogenized. In the case of magnetic sensor particles, an additional $5 \mathrm{mg}$ of magnetite nanoparticles (EMG1300) were added and mixed thoroughly. After complete removal of the solvent, the blend was sintered for 4 hours in a drying furnace at $230^{\circ} \mathrm{C}$. Finally, the composite material was manually grounded in a mortar until a fine powder was obtained. The microparticles were dispersible in a 1:1 mixture of ethanol and water.

\section{Luminescence measurements - setup and fitting of the fluorescence spectrophotometer}

Fluorescence measurements were performed with a Hitachi F-7000 fluorimeter from Metrohm Inula GmbH (Vienna, Austria, http://www. metrohm.com) equipped with a red-sensitive photomultiplier tube R9876 (PMT) from Hamamatsu Photonics (Herrsching am Ammersee, Germany, http://www.hamamatsu.com). A self-made fitting (Supplementary Figure S1) was installed, enabling the excitation with a diode laser. The fitting allows for thermostatted and stirred measurements. A $120 \mathrm{~mW}$ laser diode with a peak emission at 980 $\mathrm{nm}$, an NIR interference filter ( $980 \mathrm{~nm}$, FWHM: $10 \mathrm{~nm}$ ) from Melles Griot GmbH (Bensheim, Germany, www.cvimellesgriot.com), and a collimator lens were used for luminescence measurements.

Oxygen partial pressure was adjusted using a gas mixing device of Vögtlin Instruments (Aesch, Switzerland, www.red-y. com), nitrogen gas, and compressed air. The gas mixing device was triggered by a self-made LabVIEW code.

\section{Lifetime measurements}

The setup (Supplementary Figure S2) consisted of $980 \mathrm{~nm} \mathrm{CW}$ laser module $\left(200 \mathrm{~mW}, 130 \mathrm{~W} \mathrm{~cm}{ }^{-2}\right.$ ) from Picotronic (Koblenz, Germany, www.picotronic.com) and an optical chopper (MC2000 with two-slot chopper blade MC1F2) from Thorlabs (Dachau, Germany, www.thorlabs.com). We used optical bandpass filters (FF01-535 and FF01-665/150/25) from Semrock (Rochester, NY, USA, www.semrock.com) for measuring single emission bands. The signal was amplified using a photomultiplier unit (PMT) from PreSens (Regensburg, Germany, www.presens.com) and analyzed using a digital storage oscilloscope (DSO 8204) from Voltcraft (Wollerau, Switzerland, www.voltcraft.ch) in combination with a home-made LabVIEW code.

\section{Transmission electron microscopy}

Upconverting nanoparticles have been characterized by TEM studies performed by using a $120 \mathrm{kV}$ Philips CM12 microscope from FEI (Hillsboro, OR, USA, www.fei.com).

\section{RESULTS AND DISCUSSION}

The composition and preparation of sensor particles are schematically shown in Figure 1a. We used PPSQ as a sensor matrix. This ladder-like polymer is used in electronics as a semiconductor, insulator, or organic light emitting diode material, as well as a catalyst for chemical reactions 32,33 . Its properties, including an amorphous structure and appropriate oxygen permeability, are 


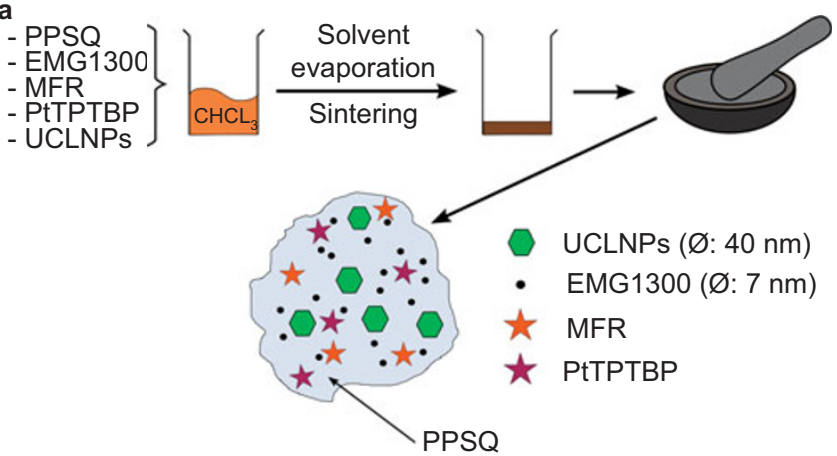

b

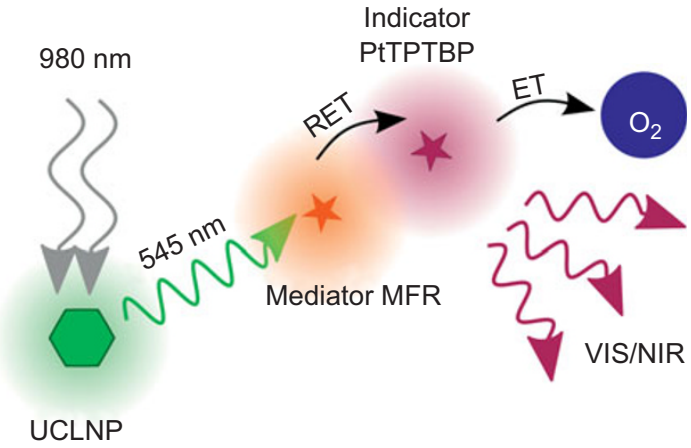

Figure 1 (a) Composition and preparation of sensor particles are shown schematically. The matrix material (PPSQ), magnetic nanoparticles (EMG1300), mediator dye (Macrolex Fluorescent Red G, MFR), indicator dye (PtTPTBP), and UCLNPs were swollen, dissolved, and dispersed in $\mathrm{CHCl}_{3}$. After homogenization, the solvent was evaporated. The dry material was then sintered for 4 hours at $230^{\circ} \mathrm{C}$. Microparticles were achieved by manually grinding the bulk material. (b) Scheme showing the light cascade inside a sensor particle. A $980 \mathrm{~nm}$ laser light excites the UCLNPs. The emitted light is absorbed by the mediator dye and subsequently non-radiatively transmitted to the indicator dye. Its luminescence is dynamically quenched by molecular oxygen.

similar to those of ormosil glasses, which have been used for optical oxygen sensors. This polymer enables easy preparation of sensor layers and particles by mixing and sintering of the components.

PtTPTBP, used as an indicator, offers similar sensitivity toward oxygen as the platinum complex of the widely used tetrakis- (pentafluorophenyl)porphyrin, but possesses better photophysical properties, such as higher photostability, molar absorbance, and quantum yield. Another advantage is its emission above 720 $\mathrm{nm}$ with a peak maximum at $770 \mathrm{~nm}$, which is not absorbed by biological matter. In principle, this approach could be suitable for subcutaneous in vivo measurement of oxygen because the NIR excitation as well as the far-red emission efficiently penetrate into the tissue up to a few millimetres. To excite PtTPTBP by NIR via UCLNPs, a mediator is necessary as shown in Figure $1 \mathrm{~b}$. This mediator dye, MFR, does not respond to changes in oxygen concentration and has the potential to pass the energy of the UCLNP emission to the indicator dye. The mediator dye absorbs the green emission of the UCLNPs and further excites the oxygen-sensitive dye, PtTPTBP, via non-radiative energy transfer. The emission intensity of PtTPTBP, which is dependent on the oxygen partial pressure in the sample, can then be monitored. Spectral properties of the employed dyes are shown in Supplementary Figure S3.

UCLNPs are known for their low-quantum yield ${ }^{34}$; therefore, the most important step in the light cascade is the absorbance of the upconversion emission by the mediator. This step was investigated using PPSQ particles with embedded UCLNPs. Figure 2 a shows the normalized upconversion luminescence spectra of particles with and without MFR. In the presence of MFR, the green emission of the UCLNPs is significantly reduced and a broad MFR emission peak approximately $600 \mathrm{~nm}$ appears. We assume that MFR is excited via absorption of the green light via radiative energy transfer. This assumption is based on two experimental findings: first, the green emission of the UCLNPs is not quenched completely despite the MFR concentration of $0.5 \%$, which is significantly higher than the $\mathrm{Er}$ concentration of $0.2 \%$ in the particles. In the case of resonance energy transfer (RET), for such high acceptor concentrations, a stronger reduction of the green UCLNP peak is expected. Note that a higher concentration of MFR did not result in stronger quenching of the green emission. Second, the emission peak of MFR is weak. By excitation via RET, the peak area of the MFR emission corresponds to the reduced area of the green UCLNP emission. We have proven in previous experiments that this is not attributed to self-quenching of MFR. To exclude quenching by water, the measurements were performed in an ethanol $(E t O H) /$ deuterium oxide $\left(D_{2} O\right)$ mixture. RET between UCLNPs and MFR should change the luminescence lifetime of the green upconversion emission. However, there was no significant change in the lifetime of particles containing only $\mathrm{NaYF}_{4}(\mathrm{Yb}, \mathrm{Er})$ and particles consisting of $\mathrm{NaYF}_{4}(\mathrm{Yb}, \mathrm{Er})$ and MFR (Figure 2b). As a consequence, the mechanism for exciting the mediator by NIR excitation can be ascribed to an inner-filter effect or
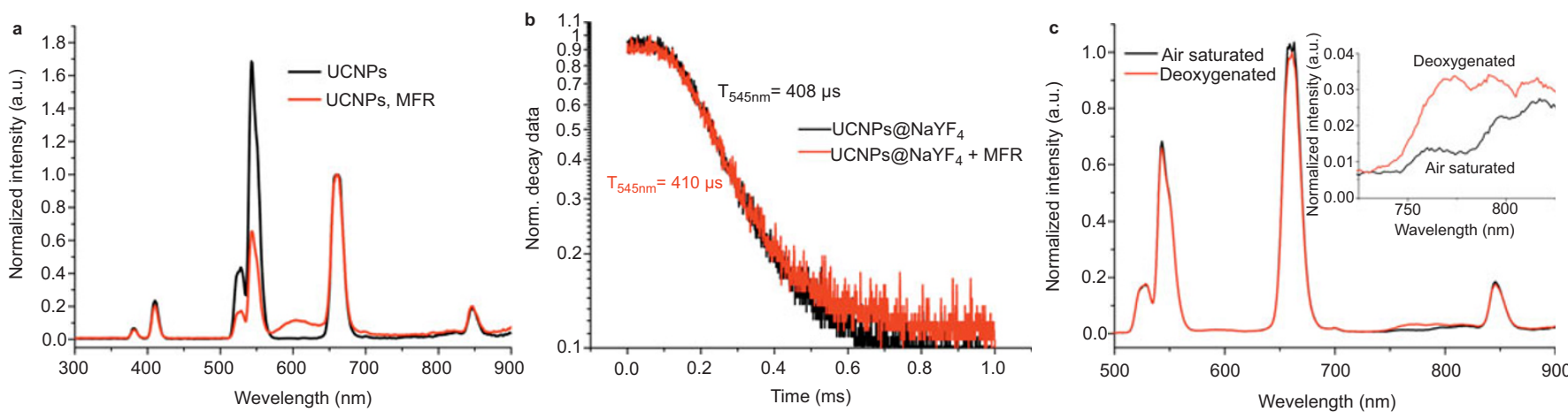

Figure 2 (a) Normalized upconversion luminescence spectra of PPSQ particles containing UCLNPs (black line) and particles containing both UCLNPs and the mediator dye MFR (red line) upon $980 \mathrm{~nm} \mathrm{CW}$ laser excitation $\left(6 \mathrm{~W} \mathrm{~cm}^{-2}\right.$ ). (b) Upconversion luminescence lifetime measurements of the green emission band UCLNPs with shell embedded in PPSQ particles in the presence (red line) and absence (black line) of the mediator dye MFR. Particles were dispersed in EtOH/D $\mathrm{D}_{2} \mathrm{O}(1: 1)$ and excited using a $980 \mathrm{~nm} \mathrm{CW} \mathrm{laser}\left(130 \mathrm{~W} \mathrm{~cm}^{-2}\right)$. (c) Normalized upconversion luminescence spectra sensor particles composed of UCLNP, mediator dye MFR, and oxygen indicator dye PtTPTBP recorded in air-saturated (black line) and deoxygenated (red line) dispersion. The inset is showing the oxygen-dependent signal of PtTPTBP between 725 $\mathrm{nm}$ and $825 \mathrm{~nm}$. (a) and (b) Particles were dispersed in a 1:1 mixture of ethanol and water. 
radiative energy transfer. For further improvement of the upconversion luminescence, the $\mathrm{NaYF}_{4}(\mathrm{Yb}, \mathrm{Er})$ crystals were coated with an inert shell of $\mathrm{NaYF}_{4}$ of approximately $7 \mathrm{~nm}$ (Supplementary Figure S4) to prevent non-radiative decay caused by surface defects and polymer surroundings. Consequently, an enhancement of the luminescence intensity of the green emission compared to $\mathrm{NaYF}_{4}$ $(\mathrm{Yb}, \mathrm{Er})$ without a shell is found ${ }^{35,36}$. In Figure 2c, the emission spectra of the sensor particles are plotted, revealing a change of luminescence intensity in aerated and deoxygenated solutions. In the absence of oxygen the green UCLNP peak is significantly less pronounced and the fluorescence of MFR vanishes completely, indicating a complete energy transfer from MFR to PtTPTBP. The inset in Figure $2 \mathrm{c}$ shows the quenching of the PtTPTBP emission by oxygen.

Significant advantages of magnetic particles include enhanced sensor signal attributed controllable accumulation of the particles, the possibility of generating in situ sensor spots at any desirable location and the possibility of easily retaining the sensor particles in a flow. In general, two opposite effects are observed by the introduction of magnetite into the sensor particles. First, for dispersed particles a decrease of the luminescence intensity by a factor of approximately 6 as a result of the high lightabsorbing properties of the magnetic nanoparticles is observed (Supplementary Figures S5a and S6). Second, collected magnetic sensor particles show an emission increase by a factor of 10 compared to dispersed sensor particles (Supplementary Figure $\mathrm{S5b}$ ). As a result, the sensor signal is enhanced by a factor of $5 / 3$. After removing the permanent magnet, the sensor particles could be re-suspended in the sample by stirring.

The response of collected magnetic sensor particles exposed to air-saturated and oxygen-free solutions is depicted in Figure 3a. The particles are magnetically collected in the focus of the $980 \mathrm{~nm}$ laser light source. The oxygen-dependent emission of PtTPTBP was detected at a wavelength of $760 \mathrm{~nm}$ to $780 \mathrm{~nm}$. A significantly higher signal to noise ratio of $~ 35$ was obtained in comparison to $\sim 13$ for non-magnetic dispersed particles (Supplementary Figure S7). Calibration curves are shown in Figure $3 \mathrm{~b}$. The magnetic sensor particles showed a good response, especially at low oxygen concentrations (up to $100 \mathrm{hPa} \mathrm{O}_{2}$ ). The calibration curve was fitted with a modified two-site model ${ }^{37}$, assuming the existence of two fractions of dye molecules within the sensor particles; one fraction is quenchable by oxygen and the other is not. $\mathrm{A} \mathrm{K}_{\mathrm{sv}}$ value of $0.0445 \mathrm{hPa}^{-1}$ was obtained. The sensitivity expressed by $I_{0} / I_{\text {air }}-1$ is 1.8. In comparison, the reported oxygen-sensing film employing UCNLPs as nanolamps by Achatz et al. showed a significantly lower sensitivity with a $\mathrm{K}_{\mathrm{SV}}$ value of $0.0101 \mathrm{hPa}^{-1}$ and a $I_{0} / I_{\text {air }}-1$ value of $0.25^{21}$. The higher sensitivity $\left(\mathrm{K}_{\mathrm{SV}}=0.0607 \mathrm{hPa}^{-1}\right)$ of nonmagnetic sensor particles is most likely attributed to the quenching effects by the incorporated magnetite nanoparticles.

\section{CONCLUSION}

In summary, we herein reported luminescence-based oxygen sensor composite particles using upconverting nanocrystals as an excitation source and a dye with far-red emission to minimize autofluorescence in biological samples. For signal enhancement, magnetic particles were embedded into the composite, enabling the collection of the sensor particles by a permanent magnet at any desired position to form an in situ sensor spot. The manually ground micrometre-sized particles were easily prepared, and smaller sizes were obtained by automated milling. An increase in the signal was achieved by incorporating a directly bound mediator dye into the particle.

In principle, this concept could be useful for applications in biological materials, such as cell cultures, tissues, or subcutaneous measurements. Both NIR excitation and far-red emission offer the advantage of good penetration efficiency into biological matter. In addition, NIR excitation generates low or practically zero
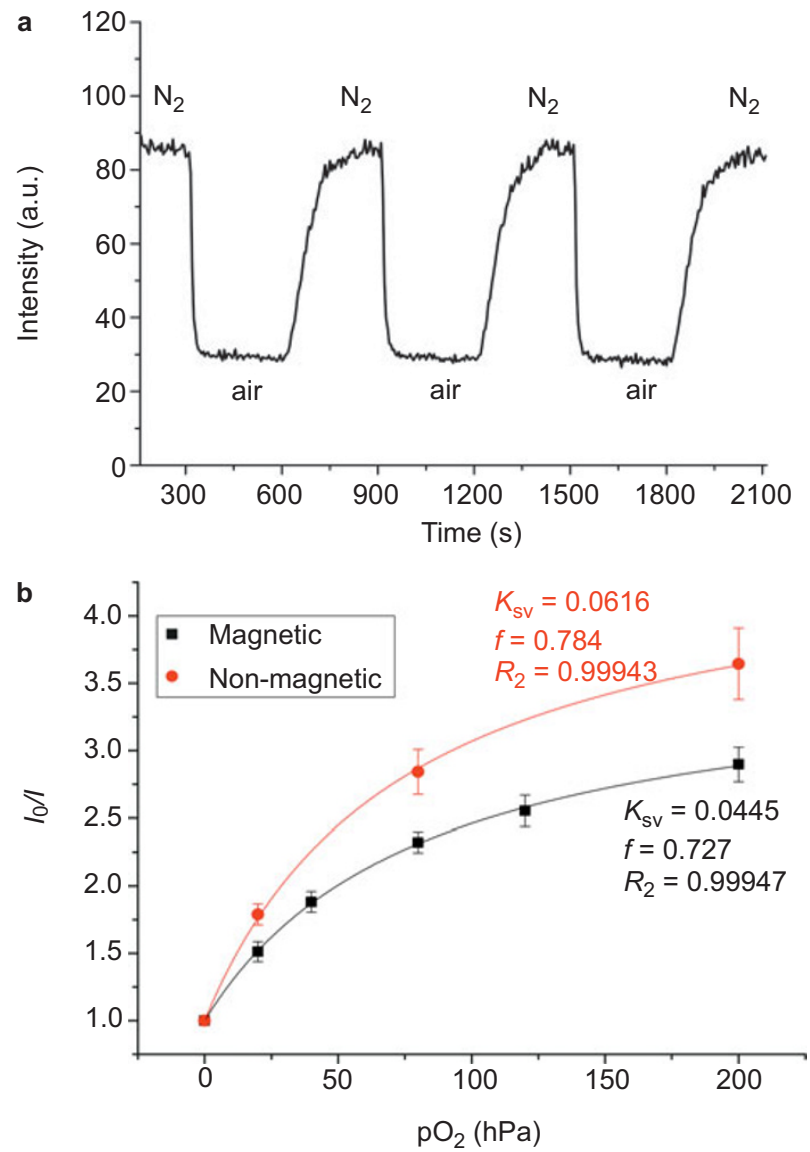

Figure 3 (a) Response curves to dissolved oxygen of magnetic sensor particles collected in the focus of the $980 \mathrm{~nm}$ laser diode beam $(6 \mathrm{~W}$ $\mathrm{cm}^{-2}$ ) using an external permanent magnet. Particles were dispersed in a 1:1 mixture of ethanol and water. (b) Calibration curves represented as Stern-Volmer plots of the luminescence intensity. The calibration data are $\mathrm{K}_{\mathrm{SV}}=0.0445, f=0.727$, and $R^{2}=0.99947$ for the magnetic particles (black squares) and $\mathrm{K}_{\mathrm{SV}}=0.0616 \mathrm{hPa}^{-1}, f=$ 0.784 , and $R^{2}=0.99943$ for the non-magnetic particles (red dots).

autofluorescence. Furthermore, UCLNP magnetic sensor particles are suggested to be applied in microfluidic systems for monitoring of miniaturized cell cultures.

\section{ACKNOWLEDGEMENTS}

This research was funded by Austrian Science Fund (FWF; I 442-N19) and Deutsche Forschungsgemeinschaft (DFG; WO 669/12-1), both within the framework of an ERA Chemistry project.

\section{COMPETING INTERESTS}

The authors declare no conflict of interest.

\section{REFERENCES}

1 Wang $X$, Wolfbeis OS. Optical methods for sensing and imaging oxygen: Materials, spectroscopies and applications. Chemical Society Reviews 2014; 43: 3666-3761.

2 Stich MIJ, Fischer LH, Wolfbeis OS. Multiple fluorescent chemical sensing and imaging. Chemical Society Reviews 2010; 39: 3102-3114.

3 McDonagh C, Burke CS, MacCraith BD. Optical chemical sensors. Chemical Reviews 2008; 108: 400-422.

4 Ungerböck B, Pohar A, Mayr T et al. Online oxygen measurements inside a microreactor with modeling of transport phenomena. Microfluidics and Nanofluidics 2013; 14: 565-574. 
5 Fercher A, Borisov SM, Zhdanov AV et al. Intracellular $\mathrm{O} 2$ sensing probe based on cell-penetrating phosphorescent nanoparticles. ACS Nano 2011; 5 5499-5508.

6 Borisov SM, Mayr T, Klimant I. Poly(styrene-block-vinylpyrrolidone) beads as a versatile material for simple fabrication of optical nanosensors. Analytical Chemistry 2008; 80: 573-582.

7 Lee YEK, Kopelman R. Optical nanoparticle sensors for quantitative intracellular imaging. Wiley Interdisciplinary Reviews: Nanomedicine Nanobiotechnology 2009; 1: 98-110.

8 Borisov SM, Klimant I. Optical nanosensors-Smart tools in bioanalytics. The Analyst 2008; 133: 1302-1307.

9 Baù L, Tecilla P, Mancin F. Sensing with fluorescent nanoparticles. Nanoscale 2011; 3: 121-133.

$10 \mathrm{Xu}$ CT, Zhan Q, Liu H et al. Upconverting nanoparticles for pre-clinical diffuse optical imaging, microscopy and sensing: Current trends and future challenges. Laser \& Photonics Reviews 2013; 7: 663-697.

11 Haase M, Schäfer H. Upconverting nanoparticles. Angewandte Chemie International Edition 2011; 50: 5808-5829.

12 Auzel F. Upconversion and anti-Stokes processes with $\mathrm{f}$ and $\mathrm{d}$ ions in solids. Chemical Reviews 2004; 104: 139-174.

$13 \mathrm{Gu}$ Z, Yan L, Tian G et al. Recent advances in design and fabrication of upconversion nanoparticles and their safe theranostic applications. Advanced Materials 2013; 25: 3758-3779.

14 Gnach A, Bednarkiewicz A. Lanthanide-doped up-converting nanoparticles: Merits and challenges. Nano Today 2012; 7: 532-563.

15 Gorris HH, Wolfbeis OS. Photon-upconverting nanoparticles for optical encoding and multiplexing of cells, biomolecules, and microspheres. Angewandte Chemie International Edition 2013; 52: 3584-3600.

16 Ge X, Dong L, Sun L et al. New nanoplatforms based on UCNPs linking with polyhedral oligomeric silsesquioxane (POSS) for multimodal bioimaging. Nanoscale 2015; 7: 7206-7215.

17 Wilhelm S, del Barrio M, Heiland J et al. Spectrally matched upconverting luminescent nanoparticles for monitoring enzymatic reactions. ACS Applied Materials \& Interfaces 2014; 6: 15427-15433.

18 del Barrio $M$, de Marcos S, Cebolla $V$ et al. Enzyme-induced modulation of the emission of upconverting nanoparticles: Towards a new sensing scheme for glucose. Biosensors and Bioelectronics 2014; 59: 14-20.

19 Ali R, Saleh SM, Meier RJ et al. Upconverting nanoparticle based optical sensor for carbon dioxide. Sensor and Actuators B: Chemical 2010; 150: 126-131.

20 Sun LN, Peng $\mathrm{H}$, Stich MIJ et al. pH sensor based on upconverting luminescent lanthanide nanorods. Chemical Communication 2009; 33: 5000-5002.

21 Achatz DE, Meier RJ, Fischer LH et al. Luminescent sensing of oxygen using a quenchable probe and upconverting nanoparticles. Angewandte Chemie International Edition 2011; 50: 260-263.

22 Liu J, Liu Y, Liu Q, et al. Iridium(III) complex-coated nanosystem for ratiometric upconversion luminescence bioimaging of cyanide anions. Journal of the American Chemical Society 2011; 133: 15276-15279.
23 Zhang J, Li B, Zhang $\mathrm{L}$ et al. An optical sensor for $\mathrm{Cu}(\mathrm{II})$ detection with upconverting luminescent nanoparticles as an excitation source. Chemical Communication 2012; 48: 4860-4862.

24 Esipova TV, Ye X, Collins JE et al. Dendritic upconverting nanoparticles enable in vivo multiphoton microscopy with low-power continuous wave sources. Proceedings of the National Academy of Sciences of the USA 2012; 109: 20826-20831.

25 Arppe R, Näreoja T, Nylund $\mathrm{S}$ et al. Photon upconversion sensitized nanoprobes for sensing and imaging of $\mathrm{pH}$. Nanoscale 2014; 6: 6837-6843.

26 Liu Q, Peng J, Sun L et al. High-efficiency upconversion luminescent sensing and bioimaging of $\mathrm{Hg}$ (II) by chromophoric ruthenium complex-assembled nanophosphors. ACS Nano 2011; 5: 8040-8048.

27 Ge X, Sun L, Dang S et al. Mesoporous upconversion nanoparticles modified with a $\mathrm{Tb}$ (III) complex to display both green upconversion and downconversion luminescence for in vitro bioimaging and sensing of temperature. Microchimica Acta 2015; 182: 1653-1660.

28 Wilhelm S, Hirsch T, Patterson WM, Scheucher E et al. Multicolor upconversion nanoparticles for protein conjugation. Theranostics 2013; 3: 239-248.

29 Ungerböck B, Fellinger S, Sulzer $P$ et al. Magnetic optical sensor particles: A flexible analytical tool for microfluidic devices. Analyst 2014; 139: 2551-2559.

30 Borisov SM, Klimant I. Efficient metallation in diphenylether-A convenient route to luminescent platinum(II) complexes. Dyes and Pigments 2009; 83: 312-316.

31 Li Z, Zhang Y, Jiang S. Multicolor core/shell-structured upconversion fluorescent nanoparticles. Advanced Materials 2008; 20: 4765-4769.

32 Hong EY, Lee SH, Lee DK et al. Polyaniline films doped with ladder-type sulfonated polyphenylsilsesquioxane and unusual dependence of their electrical conductivity on temperature. Journal of Materials Chemistry 2012; 22: 18151-18155.

33 Kang DW, Kim ST, Kim YM. Preparation and characteristics of polyphenylsilsesquioxane-b-polyurethane copolymer as a dielectric material. Journal of Inorganic Organometallic Polymers 2003; 13: 157-170.

34 Boyer JC, van Veggel FCJM. Absolute quantum yield measurements of colloidal NaYF4: Er3+, Yb3+ upconverting nanoparticles. Nanoscale 2010; 2: 1417-1419.

35 Wang F, Wang J, Liu X. Direct evidence of a surface quenching effect on sizedependent luminescence of upconversion nanoparticles. Angewandte Chemie International Edition 2010; 49: 7456-7460.

36 Chen G, Shen J, Ohulchanskyy TY et al. ( $\alpha$-NaYbF4:Tm3+)/CaF2 core/shell nanoparticles with efficient near-infrared to near-infrared upconversion for high-contrast deep tissue bioimaging. ACS Nano 2012; 6: 8280-8287.

37 Carraway ER, Demas JN, DeGraff BA. Luminescence quenching mechanism for microheterogeneous systems. Analytical Chemistry 1991; 63: 332-336.

(c) This work is licensed under a Creative Commons Attribution 4.0 Unported License. The images or other third party material in this article are included in the article's Creative Commons license, unless indicated otherwise in the credit line; if the material is not included under the Creative Commons license, users will need to obtain permission from the license holder to reproduce the material. To view a copy of this license, visit http://creativecommons. org/licenses/by/4.0

Supplementary information for this article can be found on the Microsystems \& Nanoengineering website (http://www.nature.com/micronano). 